\title{
Mechanisms regulating PD-L1 expression on tumor and immune cells
}

\footnotetext{
* Correspondence: stopali1@jhmi.edu

Presented in part at the SITC $33^{\text {rd }}$ Annual Meeting, Washington DC,

November 10, 2018

${ }^{\dagger}$ George A. Crabill and Theresa S. Pritchard contributed equally to this work.

${ }^{1}$ Department of Surgery, Johns Hopkins University School of Medicine,

Sidney Kimmel Comprehensive Cancer Center, and Bloomberg Kimmel

Institute for Cancer Immunotherapy, Baltimore, MD 21287, USA

Full list of author information is available at the end of the article
}

(c) The Author(s). 2019 Open Access This article is distributed under the terms of the Creative Commons Attribution 4.0 International License (http://creativecommons.org/licenses/by/4.0/), which permits unrestricted use, distribution, and reproduction in any medium, provided you give appropriate credit to the original author(s) and the source, provide a link to the Creative Commons license, and indicate if changes were made. The Creative Commons Public Domain Dedication waiver (http://creativecommons.org/publicdomain/zero/1.0/) applies to the data made available in this article, unless otherwise stated. 


\section{Background}

Programmed death ligand 1 (PD-L1, CD274) expressed on tumor and/or immune cells in the tumor microenvironment (TME) interacts with PD-1 on tumor infiltrating lymphocytes, attenuating effector $\mathrm{T}$ cell responses and allowing tumors to escape immune attack [1,2]. Understanding how TME-resident cytokines and signaling pathways regulate $\mathrm{PD}-\mathrm{L} 1$ expression may provide therapeutic opportunities to mitigate PD-L1-induced intratumoral immunosuppression [3].

There are two general mechanisms by which tumor cells can express PD-L1, protecting them from immune elimination: "innate immune resistance" and "adaptive immune resistance" [4]. Innate resistance refers to constitutive PDL1 expression on tumor cells, resulting from PDL1 gene amplification or aberrant activation of oncogenic signaling pathways. Activation of ALK/STAT3 in T cell lymphoma [5], AP-1/JAK/STAT in classical Hodgkin lymphoma (cHL) [6], the microRNA-200/ZEB1 axis in non-small-cell lung cancer (NSCLC) [7], c-jun/STAT3 in BRAF inhibitorresistant melanoma [8], and PI3K in glioma [9] have each been reported to upregulate PD-L1 expression on tumor cells. Additionally, Myc has been shown to regulate constitutive PD-L1 expression at the mRNA level in multiple tumors, such as $\mathrm{T}$ cell acute lymphoblastic leukemia, melanoma and NSCLC [10]. Recently, post-transcriptional regulation of PD-L1 has also attracted attention, with reports that cyclin-dependent kinase-4 (CDK4) and glycogen synthase kinase 3 beta (GSK3B) can promote PD-L1 protein degradation in cultured tumors $[11,12]$.

In contrast to innate resistance, adaptive immune resistance refers to PD-L1 expression on tumor or immune cells in response to inflammatory factors secreted in the TME during antitumor immune responses. While IFN-g is generally thought to be the primary $\mathrm{T}$ cell derived cytokine responsible for adaptive PD-L1 expression, we have described several additional TME-resident cytokines that can upregulate PD-L1 expression on cultured human monocytes (Monos) and/or tumor cells, including IL-1a, IL-10, IL-27 and IL-32 g [13-15]. Transcripts for IFN-g, IL-10 and IL-32 g were over-expressed in PD-L1+ compared to PD-L1(-) melanoma biopsies; in vitro, IL-10 and IL-32 g induced PD-L1 expression on Monos but not on melanoma cells [15]. IL-1a was upregulated in EpsteinBarr virus (EBV) negative PD-L1+ cHL, and IL-27 was upregulated in EBV+ PD-L1+ cHL. When combined with IFN-g, IL-1a and IL-10 further increased PD-L1 protein expression on human Monos in vitro, compared to the effects of IFN-g alone. IL-27 increased PD-L1 expression on Monos as well as dendritic cells, $\mathrm{T}$ cells, and some tumor cell lines $[14,16]$. Others have reported that the transcription factors JAK/STAT1 [17], IRF-1 [18] and NF$\mathrm{kB}$ [19], involved in inflammatory cytokine production, can contribute to IFN-g-induced PD-L1 expression on hematopoietic tumors, lung cancer, and melanoma, respectively. In a murine medulloblastoma model, the cyclin-dependent kinase CDK5 appeared to regulate IFNg-induced PD-L1 expression [20]. Overall, existing evidence suggests that PD-L1 may be differentially regulated with respect to specific signaling pathways and transcription factors in different cell types, although IFN-g appears to be a dominant cytokine driving expression of this immunosuppressive ligand.

We undertook the current study to broadly examine mechanisms underlying constitutive and cytokine-induced PD-L1 expression in four human tumor types - melanoma (MEL), renal cell carcinoma (RCC), squamous cell carcinoma of the head and neck (SCCHN), and NSCLC and to investigate the potential roles of STAT1, STAT3, and p65 activation in driving constitutive and inducible PD-L1 expression on tumor cells and Monos.

\section{Methods}

\section{Cell culture and flow cytometry}

Established cultures of human MELs, RCCs, SCCHNs, and NSCLC (Additional file 5: Table S1) were maintained in RPMI 1640 medium or DMEM with 10\% heatinactivated fetal calf serum. Human Monos were enriched by negative selection from cryopreserved peripheral blood mononuclear cells with the Pan Monocyte Isolation Kit (Miltenyi Biotec, San Diego, CA). Cells were cultured in the presence of recombinant IFN-g (100 or $250 \mathrm{IU} / \mathrm{ml}$; Biogen, Cambridge, MA), IL-1a (10 ng/ml), IL-6 (20 ng/ $\mathrm{ml})$, IL-10 (100 ng/ml), IL-27 (50 ng/ml) or IL-32 g (100 $\mathrm{ng} / \mathrm{ml}$; all R\&D Systems, Minneapolis, MN) for the indicated time periods (Additional file 6: Table S2). In some experiments, actinomycin D (ActD, $10 \mu \mathrm{g} / \mathrm{ml}$ ) or cycloheximide (CHX, $2 \mu \mathrm{g} / \mathrm{ml}$; both Thermo Fisher Scientific, Waltham, MA) was added to cultures $1 \mathrm{~h}$ before IFN-g treatment. Adherent cells were harvested with trypsin. To assess cytokine effects on PD-L1 expression, cells were stained with anti-human PD-L1 (clone MIH4, ThermoFisher Scientific, Carlsbad, CA) or an isotype control. HLA-DR (clone L243, Becton Dickenson, San Jose, CA) staining was performed simultaneously to provide a control for the effects of IFN-g. PD-L2 was stained with clone MIH18 (Thermo Fisher Scientific). Data were acquired on the BD FACSCalibur and analyzed with FlowJo Software (TreeStar, Ashland, OR). Expression level of a molecule was calculated as delta mean fluorescence intensity ( $\triangle \mathrm{MFI}$ ), which is MFI of specific staining - MFI of isotype control staining. Cytokine-induced expression of a molecule was calculated as $\Delta \Delta \mathrm{MFI}$, which is $\Delta \mathrm{MFI}$ with cytokine exposure $-\Delta$ MFI without cytokine exposure.

Real time quantitative reverse transcriptase PCR (qRT-PCR) mRNA was extracted from cells $6-16 \mathrm{~h}$ after cytokine treatment with the RNeasy Mini Kit (QIAGEN, Germantown, 
MD). Total mRNA from each sample was reversetranscribed with the $\mathrm{qScript}^{\mathrm{Tm}}$ cDNA SuperMix (Quanta Bioscience, Beverly, MA). Real-time PCR was performed in triplicate for each sample using commercial primers and probes for CD274, HLA-DRA, and housekeeping genes (Thermo Fisher Scientific). Forty cycles of PCR were conducted using a QuantStudio 12 K Flex Real-Time PCR System. Results were analyzed using the manufacturer's software (Applied Biosystems). Fold change of mRNA expression before and after cytokine treatment was calculated as $2^{\wedge}(\Delta \mathrm{Ct}$ before $-\Delta \mathrm{Ct}$ after $)$, in which $\Delta \mathrm{Ct}=\mathrm{Ct}_{\text {specific probe }}-$ $\mathrm{Ct}$ internal control.

\section{Western blotting}

Lysates of whole cells or nuclear proteins were prepared with M-Per and NE-Per (Thermo Fisher Scientific) respectively, as described [15]. Briefly, $20 \mu \mathrm{g}$ protein per lane was separated by 4-12\% Bis-Tris SDS-PAGE under reducing conditions and transferred to a polyvinylidene difluoride membrane, which was blocked with 5\% dry non-fat milk. Membranes were stained with antibodies specific for signal transducer and activator of transcription (STAT)1 (polyclonal, catalog \# 9172), phospho-STAT1 (clone 58D6), STAT3 (clone 124H6), phospho-STAT3 (pSTAT3; clone M9C6), p65 (clone D14E12), phospho-p65 (pp65; clone 93H1), c-jun (clone 60A8) and phospho-c-jun (pcjun; clone D47G9) (all Cell Signaling Technology, Beverly, MA) at $4{ }^{\circ} \mathrm{C}$ overnight. Membranes were counterstained with anti-rabbit IgG-HRP (1:1000-1:12,000 dilution) or anti-mouse IgG-HRP (1:1000-1:5000) for $1 \mathrm{~h}$ at room temperature (GE Healthcare, UK or Kindle Bioscience, Greenwich, CT). Blots were also stained with anti-betaactin-peroxidase (1:200,000 dilution; Sigma, St. Louis, MO, clone AC-15). Proteins were detected by ECL Western blotting detection reagents (GE Healthcare) or $\mathrm{Hi} / \mathrm{Lo}$ Digital-ECL Western Blot Detection Kit (Kindle Bioscience) and the density of the target molecule was quantified with the ImageJ program (https://imagej.nih.gov/ij/) [21]. Normalized density was calculated as the ratio of target molecule density to beta-actin density.

\section{Short inhibitory RNA (siRNA) transfection}

ON-TARGET plus SMART pool siRNAs for STAT1, STAT3, and p65 were purchased from Dharmacon (Lafayette, CO). siRNA transfection was done with the Nucleofector II or 4D-nucleofector device (Lonza, Basel, Switzerland) following the Amaxa Cell Line Nucleofector Kit, Human Monocyte Nucleofector Kit, or SF/SE Cell Line 4D Nucleofector X kit protocols. Briefly, $1 \times$ $10^{6}-4 \times 10^{6}$ tumor cells or $1 \times 10^{7}$ Monos were suspended in $100 \mu \mathrm{l}$ transfection solution supplemented with 100-300 pmol specific or scrambled siRNA. Electroporation was done with transfection programs recommended in the Lonza Knowledge Center (https:// knowledge.lonza.com/) [22]. Two days after transfection, cells were incubated with cytokines. Knockdown effects and transcription factor phosphorylation were detected 15 min later by Western blotting. Percentage of knockdown was calculated based on the actin-normalized density of the target molecule in Western blotting, by the formula (scrambled siRNA - specific siRNA)/scrambled siRNA $\times 100$. The average targeted knockdown achieved in this study was 70\%. PD-L1 and HLA-DR expression at the cell surface was detected and quantified $24 \mathrm{~h}$ later by flow cytometry, and the effects of knockdown with target-specific siRNAs were calculated with reference to scrambled siRNA.

\section{PDL1 promoter region sequencing}

Genomic DNA from cultured tumor cell lines or cryopreserved peripheral blood lymphocytes was extracted from $1 \times 10^{6}$ cells using the PureLink Genomic DNA kit (Thermo Fisher Scientific, K1820-00). Based on the public PDL1 (CD274) gene sequence (GenBank NC_ 000009.12), three primers (PDLP-F1, 5'GTTTCCAGGCATCACCAGATGCT; PDLP-F2, 5’TCCTCATGGGTTATGTGTAGTTTG; PDLP-R, 5'CCTCATCTTTCTGGAATGCCCTA) were designed to amplify $2.1 \mathrm{~kb}$ and $1.1 \mathrm{~kb}$ regions that are immediately upstream of the ATG translation start site. These two regions were amplified using an Expand TM High Fidelity PCR system (Sigma, catalog \# 11732650001). Amplified PCR products were purified by a QIAquick PCR Purification kit (Qiagen, catalog \# 28104) and sent to the Johns Hopkins University Core Facility for Sanger sequencing. Amplicons were sequenced using the following primers: PDLP-seq, 5'TGCTGAATTCAGTCCTTAATGG and PDLP-seqR, 5'CCATTAAGGACTGAATTCAGCA; PDLP-seq2, 5'CAGATACTCTGGAAGAGTGGCT and PDLP-seq2R, 5’AGCCACTCTTCCAGAGTATCTG.

\section{Results}

IFN-g-induced PD-L1 protein expression on tumor cells is associated with de novo PD-L1 (CD274) mRNA transcription

We first assessed constitutive tumor cell surface PD-L1 protein expression with flow cytometry on 32 established tumor lines, including 17 MELs, 11 RCCs, 3 SCCHNs and 1 NSCLC. PD-L1 was not constitutively expressed on 16 of 17 cultured MELs, nor on one NSCLC; in contrast, 8 of 11 RCCs and 3 of 3 SCCHNs constitutively expressed PD-L1 on the cell surface (Fig. 1a). The absence of constitutive expression on melanoma cell lines contrasts with a previous report [23]. Regardless of baseline PD-L1 expression, all four tumor types showed significantly enhanced PD-L1 protein expression after brief exposure to IFN-g $(p<0.0001$; Fig. $1 \mathrm{~b}$ and c) [15]. Cell surface expression of CD119 (IFN-g receptor 1), the 




ligand-binding alpha chain of the heterodimeric IFN-g receptor, was assessed with flow cytometry on 28 of 32 cell lines and was compared to IFN-g-enhanced PD-L1 protein expression. Although CD119 was detected in each cell line, CD119 levels did not correlate with the magnitude of increased PD-L1 expression after IFN-g exposure (Spearman correlation test, $\mathrm{r}=0.19, p=0.32$; data not shown), suggesting that even low levels of CD119 are sufficient for signal transduction. To investigate whether induction of PD-L1 protein was associated with new synthesis of $P D L 1$ mRNA, changes in mRNA and protein levels were examined in 32 tumor cell lines representing four cancer types, before and after IFN-g treatment. Changes in PDL1 mRNA expression correlated significantly with PD-L1 cell surface protein expression $(p<0.0001$; Fig. 1d). These results suggest that IFN-g activates factors promoting new PDL1 mRNA transcription. In some cell lines, IFN-g also induced or enhanced tumor cell surface expression of PD-L2, the second ligand for PD-1, although these levels were substantially lower than for PD-L1 (Additional file 7: Table S3).

To further explore this phenomenon, we incubated cultured MELs with ActD, a mRNA transcription inhibitor, or $\mathrm{CHX}$, a protein synthesis inhibitor, prior to IFN-g exposure. Six h after IFN-g exposure, we found that each chemical completely blocked the emergence of PD-L1 protein on the cell surface. As expected, in the same cells, ActD suppressed IFNg-induced PDL1 mRNA transcription while $\mathrm{CHX}$ did not (Additional file 1: Figure S1). These data suggest that IFN-g drives new PD-L1 
transcription and translation, and that translocation of preexisting intracellular PD-L1 protein stores is not a major mechanism underlying IFN-g-induced PD-L1 expression on the cell surface.

\section{STAT1 but not STAT3 mediates IFN-g-induced PD-L1 protein expression on tumor cells}

IFN-g is known to signal through the transcription factor STAT1 [24]. However, STAT3 phosphorylation after binding of IFN-g to its receptor has also been reported [25]. To evaluate the potential roles of STAT1 and/or STAT3 activation in mediating PD-L1 protein expression, 31 tumor cell lines (16 MELs, 12 RCCs, $3 \mathrm{SCCH}$ Ns) were treated with IFN-g or IL-6, a prototypical STAT3 activator, and then assessed for STAT1 and STAT3 phosphorylation by Western blotting. Results showed that IFN-g induced substantial STAT1 and minor STAT3 phosphorylation in these cultured tumors $(p<0.0001$ and $p<0.0018$, respectively). Conversely, IL6 induced substantial STAT3 and minor STAT1 phosphorylation in the same cell lines $(\mathrm{p}<0.0001$ and $p<$ 0.0101 , respectively) (Fig. 2a). However, in contrast to IFN-g, IL-6 induced minimal PD-L1 protein expression on only 2 of 32 tumor lines tested (not shown). To further explore the potential roles of STAT1 and STAT3 in IFN-g-induced PD-L1 expression on tumor cells, we knocked down their expression with specific siRNAs. STAT1, but not STAT3 knockdown reduced IFN-ginduced PD-L1 protein expression by $32-70 \%$ in 6 cell lines tested ( 2 representative examples are shown in Fig. 2b-e). Notably, constitutive PD-L1 expression was not affected by STAT1 or STAT3 knockdown in two SCCHNs and three RCCs tested (a representative example is shown in Fig. 2e, "no cytokine" condition), suggesting that constitutive PD-L1 expression is sustained by mechanisms distinct from cytokine-induced expression. HLA-DR, another IFN-g-inducible molecule, was used as a control in these experiments. Among a total of 6 tumor cell lines assessed, which all showed reduction of IFN-g-induced PD-L1 expression after STAT1 knockdown, three also showed reduction of IFN-g-induced HLA-DR expression, regardless of baseline HLA-DR expression (e.g., JHU-022, Fig. 2e). None showed reduction of constitutive HLA-DR expression (e.g., 397mel, Fig. 2c). This is consistent with the notion that mechanisms regulating IFN-g-induced PD-L1 and HLA-DR expression are only partially overlapping.

IL-1a and IL-27 induce PD-L1 expression on tumor cells, associated with new PD-L1 mRNA transcription

We previously reported that IL-1a and IL-27 can independently induce PD-L1 protein expression on shortterm cultured human Monos [14]. In the current study, we tested the ability of these cytokines to induce PD-L1 on tumor cells. Both IL-1a and IL-27 independently and significantly enhanced or induced PD-L1 protein expression on some cultured tumor cell lines, and further increased IFN-g-induced PD-L1 expression in some cases (Fig. 3a \& c, and Fig. 3b \& d, respectively; Additional file 8: Table S4). IL-1a increased PD-L1 protein expression by $\geq 5$ MFI in 6 of 14 tumor cell lines tested; notably, the effect of combining IL-1a with IFN-g was more than additive in 12 of 14 tumor cell lines, suggesting the cooperation of distinct signaling pathways (Additional file 8: Table S4). In contrast, while IL-27 alone increased PD-L1 expression by $\geq 5$ MFI in a greater number of cell lines than did IL-1a (11 of 14), the effect of combining IL-27 with IFN-g exceeded IFN-g alone in only 7 cases and was more than additive in only one instance, suggesting that IL-27 and IFN-g signal through a shared pathway (Additional file 8: Table S4). To investigate the selective effects of IL-1a and IL-27 on certain tumor cell lines, we quantified mRNA expression for the subunits of the IL-1a (IL1R1, IL1RAP) and IL-27 receptors (IL27RA, IL6ST). Expression of these subunits was generally robust among 9 tumor cell lines tested and did not significantly correlate with cytokine-enhanced PD-L1 protein levels $(p \geq 0.05$, Spearman correlation test; data not shown), suggesting the importance of downstream events in driving PD-L1 expression.

Similar to our findings with IFN-g, changes in PD-L1 protein expression induced by IL-1a or IL-27 corresponded with changes in PDL1 gene expression, in 2 of 2 RCC lines tested (Fig. 3e). This suggests that new mRNA transcription mediated by IL-1a or IL-27 exposure contributes to PD-L1 regulation. In contrast to the findings described above, the Th17 cytokines IL-17A and IL-23, which we previously detected in the microenvironment of some human cancers but which did not enhance PD-L1 protein expression on Monos [14], also failed to induce PD-L1 on tumor cells (not shown).

\section{p65 and STAT1 respectively mediate IL-1a- and IL-27- induced PD-L1 expression on tumor cells}

To evaluate transcription factors potentially mediating the induction of PD-L1 by IL-1a and IL-27, we assessed phosphorylation of STAT1, STAT3, p65 and c-jun [26, 27]. IL27 activated STAT1 and STAT3 robustly and equivalently in two RCC cell lines tested, unlike IFN-g which preferentially activated STAT1, and IL-1a which did not activate either transcription factor (Fig. 4a). However, only STAT1 but not STAT3 siRNA knockdown impeded IL-27induced PD-L1 protein expression (Fig. 4b), consistent with previous reports examining $\mathrm{T}$ cells and ovarian cancers $[16,27]$. Using the same 14 tumor cell lines that were assessed for the effects of IL-1a and IL-27 on PD-L1 expression as shown in Fig3a and b, respectively, we tested the effects of these cytokines on transcription factor 
A
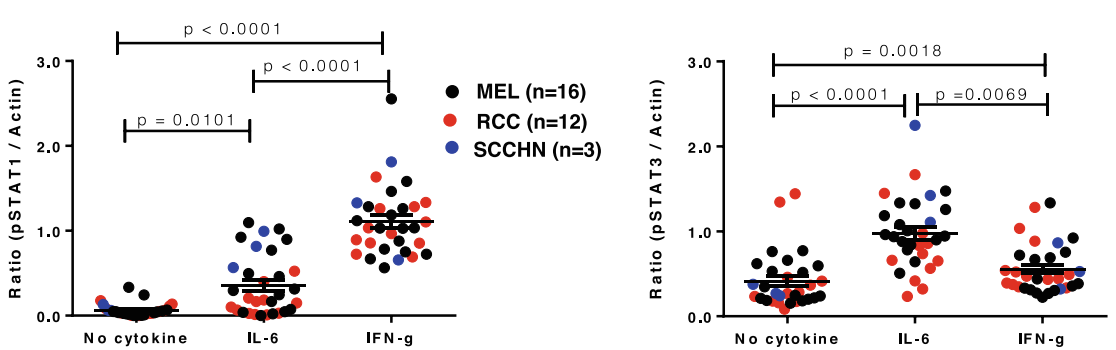

B

$397 \mathrm{mel}$

SIRNA: Scrambled STAT1 STAT3 STAT1+3

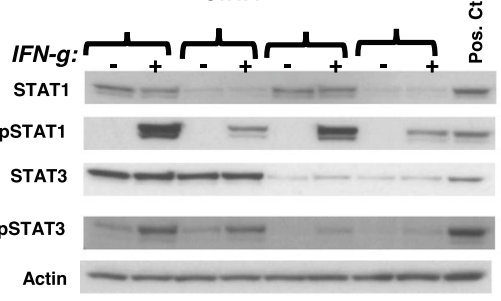

C

$397 \mathrm{mel}$

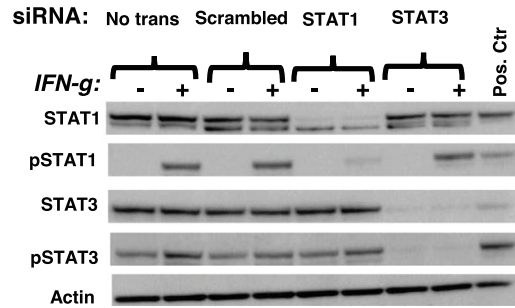

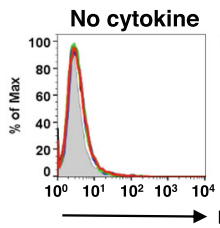
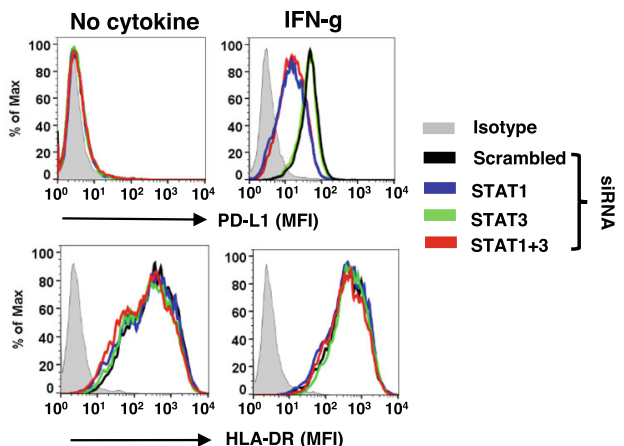

Fig. 2 STAT1, but not STAT3 phosphorylation is necessary for IFN-g-induced PD-L1 protein expression on tumor cells. a. IFN-g had a major effect on STAT1 phosphorylation (left panel) but only a minor effect on STAT3 phosphorylation (right panel) in 31 tumor cell lines tested, including MELS, RCCs, and SCCHNs. IL-6 had a reciprocal effect in the same cell lines. Cultured cells were treated with IFN-g $250 \mathrm{U} / \mathrm{ml}$ or IL-6 $20 \mathrm{ng} / \mathrm{ml}$. Cells were harvested after $15 \mathrm{~min}$ and phosphorylation of STAT1 and STAT3 was detected by Western blotting. Protein bands were quantified by ImageJ and results were normalized to beta-actin expression. Kruskal-Wallis test (Dunn's multiple comparisons test), 2-sided $p$-values. $\mathbf{b}$ and $\mathbf{c}$. Specific siRNA knockdown of STAT1, but not STAT3 mRNA expression in 397mel cells significantly reduced total and phosphorylated STAT1 proteins and reduced IFN-g-induced cell surface PD-L1 protein expression. Cultured tumor cells were transfected with 100 pmol of the indicated siRNAs and were treated 2 days later with IFN-g $250 \mathrm{U} / \mathrm{ml}$. Total and phosphorylated STAT proteins were detected by Western blotting after 15 min of IFN-g treatment, and flow cytometry for cell surface PD-L1 was conducted 1 day later. 397mel expressed HLA-DR constitutively, and this was not affected by STAT knockdown (c). d and e. In JHU-022 cultured SCCHN cells, STAT1 knockdown reduced IFN-g-induced but not constitutive ("no cytokine") cell surface PD-L1 protein expression. IFN-g also induced HLA-DR expression on JHU-022, which was reduced by STAT1 but not STAT3 knockdown. Percentages represent reduction in total PD-L1 or HLA-DR expression with STAT1 knockdown compared to scrambled siRNA control; numbers in parentheses represent reduction in the amount of PD-L1 or HLA-DR expression that was induced by IFN-g above "no cytokine" baseline expression. Data in panels B-E are representative of 6 tumor lines (4 MELs and 2 SCCHNs). No trans, no transfection; Pos. Ctr., positive control cell lines, mixture of equal amounts of IFN-treated PC-3 cells as PSTAT1 positive control and IL-6-treated COS-7 cells as PSTAT3 positive control; Scrambled, non-specific siRNA mixture 

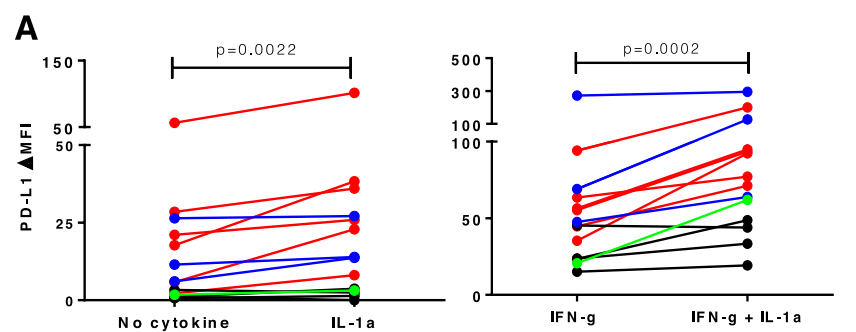

B
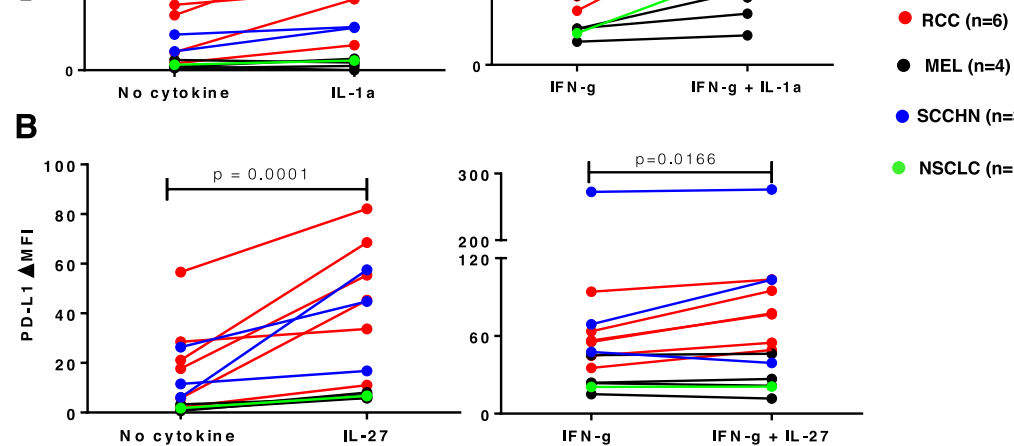

- $\operatorname{sccHN}(n=3)$

- NSCLC ( $n=1)$

C



$-1 a$



D
IL-27

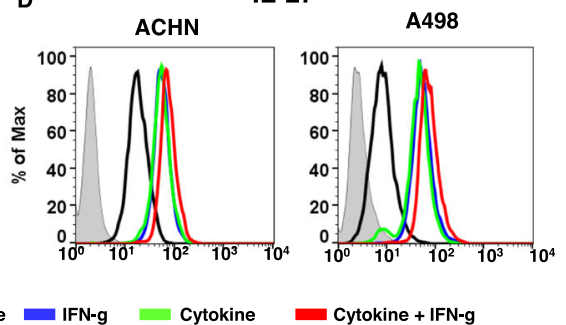

E

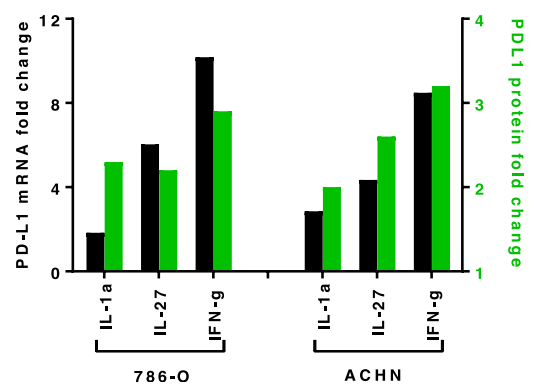

Fig. 3 IL-1a- and IL-27-induced PD-L1 protein expression are associated with new PD-L1 mRNA transcription in tumor cells. Fourteen cultured tumor lines were treated with IL-1a $(10 \mathrm{ng} / \mathrm{ml})$ or IL-27 $(50 \mathrm{ng} / \mathrm{ml})$ for $48 \mathrm{~h}$, and cell surface PD-L1 protein was detected by flow cytometry. a. IL1a alone (left panel) or in combination with IFN-g (right panel) increased PD-L1 expression on tumor cells. $\Delta \mathrm{MFI}$, mean fluorescence intensity of PD-L1 staining - isotype control staining. Wilcoxon matched-pairs signed rank test, 2-sided $p$-values. b. IL-27 independently increased PD-L1 protein expression on tumor cells (left panel), and a further increase was observed when IL-27 was combined with IFN-g (right panel). c. Overlay of flow cytometry histograms from two representative RCC cell lines (ACHN and A498). Either IL-1a or IFN-g independently increased PD-L1 expression, and a greater increase was observed when these cytokines were combined. Note that ACHN and A498 both show constitutive PD-L1 expression in the absence of cytokine treatment. d. Overlay of flow cytometry histograms of ACHN and A498 cells treated with IL-27 or IFN-g, alone or in combination. e. Increased PD-L1 protein expression induced by IL-1a or IL-27 was associated with new PDL1 mRNA transcription in 2 RCCs tested. PD-L1 mRNA and cell surface protein were measured by qRT-PCR and flow cytometry at $16 \mathrm{~h}$ or $48 \mathrm{~h}$ after cytokine exposure, respectively

activation. In contrast to IL-27 which significantly activated STAT1 and STAT3 but not p65, IL-1a activated p65 but not STAT1 or STAT3 (Fig. 4c). Interestingly, cell surface PD-L1 expression in the same tumor cells did not correlate with the level of transcriptional activation, suggesting the influence of ancillary signaling events. C-jun, another transcription factor that has been associated in the literature with IL-1a signaling [26], was not 



C
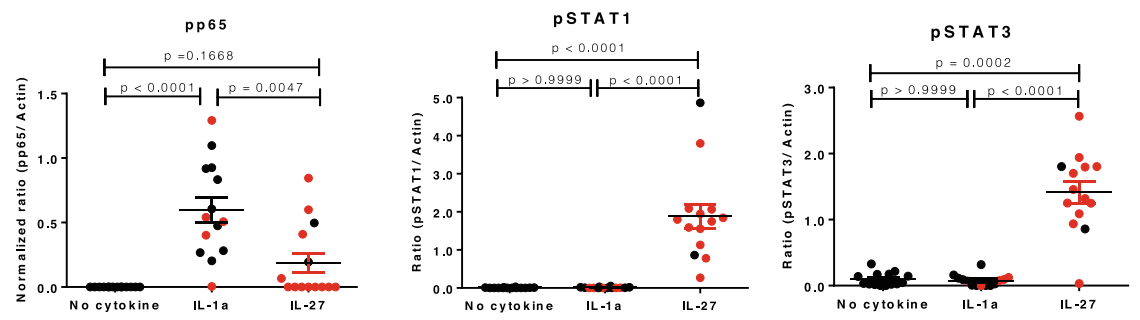

- PD-L1 not increased PD-L1 increased Total $n=14$

D

786-0

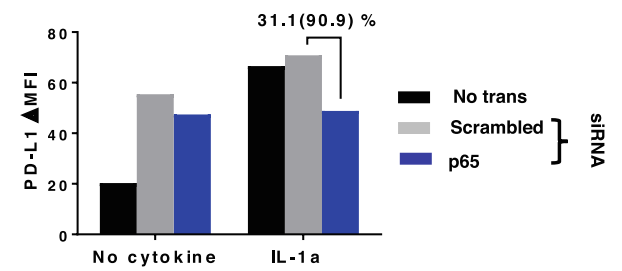

Fig. 4 p65 and STAT1 are involved in IL-1a- and IL-27-induced PD-L1 expression, respectively, in tumor cells. Cultured tumor cells were treated with IL-1a $(10 \mathrm{ng} / \mathrm{ml})$, IL-27 $(50 \mathrm{ng} / \mathrm{ml})$, or IFN-g $(100 \mathrm{IU} / \mathrm{ml})$. STAT1, STAT3, and p65 phosphorylation was detected by Western blotting $15 \mathrm{~min}$ after cytokine exposure. In experiments to inhibit phosphorylation, transcription factors first were knocked down by transfecting specific siRNAs; after 2 days, transfected cells were treated with cytokines and knockdown effects were assessed with Western blotting. PD-L1 cell surface protein expression was detected by flow cytometry 1 day after cytokine treatment. a. In two RCC cell lines, IL-27 exposure caused phosphorylation of both STAT1 and STAT3, while IFN-g selectively phosphorylated STAT1, and IL-1a did not phosphorylate either STAT1 or STAT3. Pos Ctr, positive control; mixture of equal amounts of IFN-treated PC-3 cells as a pSTAT1 positive control, and IL-6-treated COS-7 cells as a pSTAT3 positive control. b. In 397 mel, STAT1 but not STAT3 knockdown significantly reduced IL-27-induced PD-L1 expression. Results representative of 2 tumor cell lines (one MEL, one SCCHN). c. IL-1a increased p65 phosphorylation, but not STAT1 or STAT3 phosphorylation, in 14 tumor cell lines. After cytokine exposure, phosphorylation of the indicated transcription factors was detected by Western blotting. Protein bands were quantified by ImageJ and results were normalized to beta-actin expression. Because all cell lines expressed phosphorylated p65 constitutively in the absence of cytokines, values for constitutive normalized ratios have been subtracted from the data depicted for Pp65. PD-L1 increased, cytokine-induced enhancement of PD-L1 cell surface expression of $\geq 5$ MFI detected with flow cytometry (red symbols); no or lower levels of PD-L1 enhancement indicated by black symbols. Kruskal-Wallis test (Dunn's multiple comparisons test), 2-sided p-values. d. Knocking down p65 reduced IL-1a-induced PD-L1 protein expression in 786-O. Percentage represents reduction in total PD-L1 expression with p65 knockdown compared to scrambled siRNA control; number in parentheses represents reduction in the amount of PD-L1 expression that was induced by IL-1a above the "no cytokine" baseline expression. Results in panel D are representative of 3 separate experiments with 786-O. Corresponding Western blot is provided in Additional file 2: Fig. S2. $\Delta \mathrm{MFI}$, mean fluorescence of specific PD-L1 staining - isotype control staining

significantly activated in these cell lines when compared to no cytokine controls (data not shown). IL-1a-induced PD-L1 protein expression was reduced to baseline levels in the 786-O RCC line by siRNA knockdown of p65 (Fig. 4d; Additional file 2: Figure S2). However, constitutive PD-L1 expression in 786-O was not reduced by p65 knockdown (Fig. 4d, "no cytokine"). In a similar experiment with 397mel, in which IL-1a alone did not induce PD-L1 expression but was synergistic when combined with IFN-g, p65 knockdown reduced PD-L1 levels driven by the cytokine combination by $28 \%$ (data not shown). These results suggest that IL-1a signaling drives PD-L1 
protein expression through p65, but not STAT1/3, activation.

\section{PDL1 gene promoter sequence variations do not correlate with quantities of PD-L1 protein induced on tumor cells by IFN-g, IL-1a or IL-27}

To determine whether sequence variations in the promoter region of the $P D L 1$ gene, where transcription factors would be expected to bind, are associated with different levels of tumor cell PD-L1 protein expression induced by cytokines, we sequenced a $650 \mathrm{bp}$ or $2 \mathrm{~Kb}$ region upstream of the PDL1 transcription initiation codon in 33 tumor cell lines and 12 autologous normal tissues. Nine of 33 tumor cell lines harbored $-482 \mathrm{C}$ and 3 of 33 harbored $-382 \mathrm{G}$, which have been reported as SNPs (https://www.ncbi.nlm.nih.gov/snp) [28]. Neither gene alteration correlated with the level of PD-L1 protein expression induced by IFN-g, IL-1a or IL-27 exposure (Additional file 3: Figure S3).

\section{STAT1 and STAT3 play distinct roles in cytokine-induced PD-L1 expression on monocytes}

We have previously reported that IL-1a, IL-10, IL-27 and IL-32 $\mathrm{g}$ each increase PD-L1 protein expression on normal human Monos in short-term culture [13, 14]. To test if new mRNA transcription is involved in this response, PD-L1 mRNA and protein were measured in Monos after exposure to each of these four cytokines. For each cytokine tested, changes in PDL1 mRNA levels accompanied changes in PD-L1 protein expression (Fig. 5a and b). Similar to our findings in tumor cell lines, IFN-g preferentially activated STAT1 in Monos, while IL-27 activated both STAT1 and STAT3; IL-10 preferentially activated STAT3 (Fig. 5c). STAT1 and STAT3 were successfully knocked down in Monos by their respective siRNAs. Knockdown of STAT1, but not STAT3 in Monos from 2 to 4 donors reduced IFN-g- and IL-27-induced PD-L1 protein expression (Fig. 5d). Conversely, knockdown of STAT3 but not STAT1 in Monos from 4 donors reduced IL10-induced PD-L1 protein expression to constitutive levels, indicating that STAT3 mediates the effect of IL-10 in enhancing PD-L1 expression on Monos (Fig. 5d). Constitutive PD-L1 expression in monocytes was not effected by either STAT1 or STAT3 knockdown (Fig. 5d, left panel). IL-1a induced p65 phosphorylation in Monos (Additional file 4: Figure S4). However, attempted p65 knockdown in Monos was not effective, therefore, we could not assess its impact on IL-1a-induced PD-L1 protein expression. Transcription factors responsible for IL32 g-induced PD-L1 expression on Monos could not be identified, due to limited information regarding IL-32 g signaling pathways.

\section{Discussion}

There is currently heightened interest in understanding mechanisms that drive expression of the immunosuppressive ligand PD-L1 in the TME, since the PD-1:PDL1 pathway is now recognized as a dominant immune checkpoint in cancer. While this pathway has been targeted with some success in cancer therapy, current drug development strategies aim to overcome the failure of many tumors to respond to PD-1 pathway blocking drugs, and to address relapses that can occur following initial tumor regression. PD-L1 can be expressed by diverse cell types in the TME, including tumor, immune and endothelial cells. It is assumed that PD-L1 expression by any cell type in the TME can function locally to dampen antitumor immunity. This assumption has been borne out by the development of several predictive biomarkers for the therapeutic effects of anti-PD-1 drugs, that score PD-L1 protein expression on tumor cells, tumor-infiltrating immune cells, or both [29].

IFN-g secreted by tumor-reactive $\mathrm{T}$ cells, signaling through the transcription factor STAT1, is the single major cytokine that induces PD-L1 protein expression. This is associated with the phenomenon of adaptive tumor immune resistance [15]. Here we show that the effect of IFN-g in enhancing PD-L1 expression by tumor cells and Monos occurs as a result of new mRNA transcription, rather than translocation of preexisting intracellular protein stores to the cell surface. We also show that this adaptive phenomenon can increase PD-L1 expression in cells already having constitutive expression. This raises the possibility that drugs targeting STAT1 might be deployed against IFN-g-induced PD-L1 expression, to enhance anti-PD-1 therapies. Furthermore, our data indicate that targeting STAT1 might also mitigate PD-L1 expression induced by IL-27. The broad spectrum of biological roles for STAT1 suggests that it could be difficult to target this factor specifically or selectively in tumor cells. However, a recent report from Cerezo et al. suggests that drugs inhibiting eukaryotic initiation factor (eIF)4A can down-modulate STAT1 transcription in a tumor-selective manner, indirectly reducing PD-L1 expression and mediating tumor regression in murine models [30]. Further, these authors demonstrated in vitro that eIF4A chemical inhibition can decrease IFN-ginducible PD-L1 expression in cell lines from a variety of human tumor types, including melanoma, breast and colon cancer, suggesting the potential for broad applicability of this approach.

In our previous studies of the TMEs of several different cancer types, we found that elevated levels of transcripts for the cytokines IL-1a, IL-10, IL-27 and IL-32 g, in addition to IFN-g, were associated with PD-L1 protein expression. As shown in the current report, each of these cytokines can induce PD-L1 expression on tumor cells and/or Monos 
A

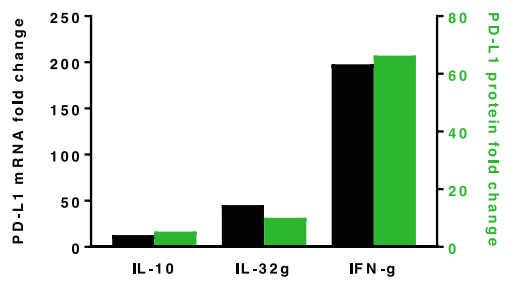

C

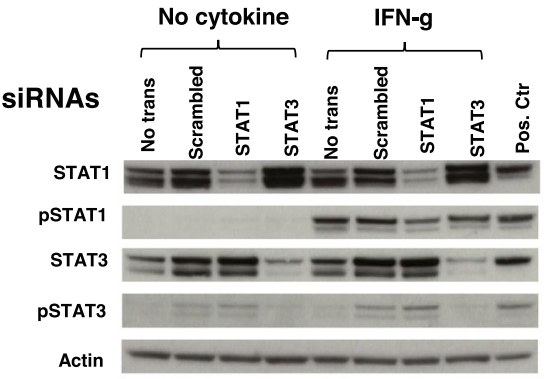

D

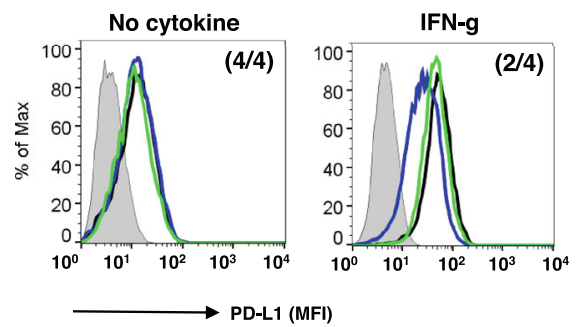



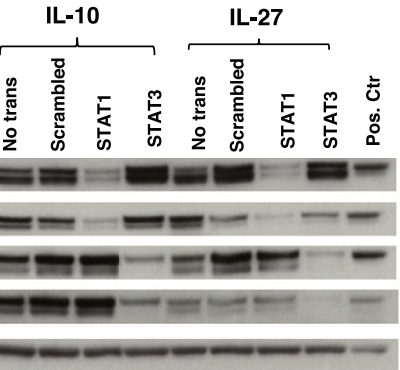

IL-10
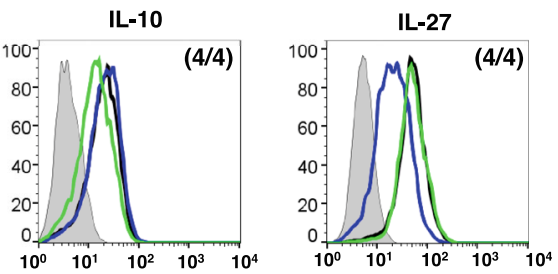

- Isotype



Fig. 5 Roles of STAT1 and STAT3 in cytokine-induced PD-L1 protein expression on monocytes. $\mathbf{a}$ and $\mathbf{b}$. Cytokine-induced PD-L1 protein expression on Monos was associated with new PDL1 mRNA transcription. Monos were treated with IL-1a, IL-10, IL-27, IL-32 g or IFN-g. PD-L1 mRNA and surface protein were measured by q-RT-PCR and flow cytometry after $16 \mathrm{~h}$ or $48 \mathrm{~h}$, respectively. Fold changes in PD-L1 protein and mRNA were calculated. Representative data from Monos derived from one of two normal donors are shown. $\mathbf{a}$. Fold changes in PD-L1 protein and mRNA levels in normal donor Monos after IL-10 (100 ng/ml), IL-32 g $(100 \mathrm{ng} / \mathrm{ml})$ or IFN-g $(100 \mathrm{IU} / \mathrm{ml})$ exposure. b. Fold changes of PD-L1 protein and mRNA levels in normal donor Monos after IL-1a $(10 \mathrm{ng} / \mathrm{ml})$, IL-27 $(50 \mathrm{ng} / \mathrm{ml})$ or IFN-g $(100 \mathrm{IU} / \mathrm{ml})$ treatment. $\mathbf{c}$ and d. Fresh isolated Monos were transfected with 300 pmol STAT1 or STAT3 siRNA and treated with the indicated cytokines 2 days later. Total or phosphorylated STATs and cell surface PD-L1 expression were assessed with Western blotting and flow cytometry after 15 min or 1 day, respectively. c. siRNA knockdown significantly reduced total and phosphorylated STAT1 and STAT3. d. STAT1 knockdown reduced IFN-g- and IL27-induced PD-L1 protein expression, while STAT3 knockdown reduced IL10-induced PD-L1 expression. Numbers in parentheses indicate number of normal donors having Monos with these findings

in vitro, although to a lesser extent than IFN-g. Furthermore, some cytokines such as IL-1a and IL-27 can have an additive or synergistic effect on PD-L1 expression when combined with IFN-g (Fig. 3, Additional file 8: Table S4). Here we show that IL-27, similar to IFN-g, induces PD-L1 by activating STAT1. However, IL-10 induces PD-L1 by activating STAT3, and IL-1a by activating the p65 transcription factor. This demonstration of the involvement of distinct signaling pathways in driving PD-L1 expression suggests new strategies for targeting diverse transcription factors, or their upstream cytokines or receptors, to mitigate
PD-L1 expression in the TME. For instance, STAT3 inhibitors, which are already in clinical testing, have been proposed to synergize with anti-PD-1/PD-L1 through their immunomodulatory effects, based on data from murine models [31]. Furthermore, because the signaling pathway by which IL-1a drives PD-L1 expression is non-overlapping with IFN-g and IL-27, our findings suggest that genetic defects in tumor cell STAT1 signaling, which can be acquired under the selection pressure of anti-PD-1 therapy [23], would not interfere with the ability of IL-1a to sustain tumor cell expression of PD-L1. Such tumors would maintain the 
ability to evade immune attack from PD-1+ T cells. Ongoing efforts to compare the immune microenvironments of tumors that are responsive or resistant to anti-PD-1 therapies will explore these hypotheses.

Finally, there appears to be a unique set of cytokines, including IL-10 and IL-32 g, which are capable of promoting PD-L1 expression on Monos but not on tumor cells, as studied in our previous report [13] and in unpublished data. The failure of tumor cells to express the IL-10 receptor may explain the failure of IL-10 to promote PD-L1 expression on them (data not shown). Regarding IL-32 g, because its receptor has not yet been identified, potential mechanisms underlying its Mono-selective PD-L1-inducing activity are unknown at this time. PD-L1 expression by Monos may be an important source of immunosuppression in the TME, and antibodies blocking cytokines or cytokine receptors mediating this expression should be considered as potential adjuncts to PD-1 pathway blockade [32].

\section{Conclusions}

Factors driving the expression of the immunosuppressive ligand PD-L1 in the TME are diverse and can vary according to cell type. Both tumor and immune cells are important sources of PD-L1 expression. Cytokines regulating PD-L1 expression, including IFN-g, IL-1a, IL-10, IL-27 and IL-32 g, signal through diverse transcription factors and have variable effects on tumor cells and Monos. Understanding the complex mechanisms underlying intratumoral PD-L1 expression will open new opportunities for developing rationally targeted combination therapies to enhance the effects of anti-PD-1 drugs.

\section{Supplementary information}

Supplementary information accompanies this paper at https://doi.org/10. 1186/s40425-019-0770-2.

Additional file 1: Figure S1. New PD-L1 mRNA and protein synthesis are required for IFN-g- induced PD-L1 surface expression on melanoma cells.

Additional file 2: Figure S2. IL-1a-induced phosphorylation of p65 is inhibited by 065 knockdown.

Additional file 3: Figure S3. Alterations in the PDL1 promoter region do not correlate with constitutive or cytokine-induced PD-L1 expression on tumor cells.

Additional file 4: Figure S4. LL-1a induces phosphorylation of p65 in monocytes from normal donors.

Additional file 5: Table S1. Thirty-four tumor cell lines used in this study.

Additional file 6: Table S2. Cytokines used in this study.

Additional file 7: Table S3. IFN-g-induced PD-L2 cell surface expression on 21 tumor cell lines.

Additional file 8: Table S4. Cytokine-induced PD-L1 expression on 14 tumor cell lines.

\section{Abbreviations}

ActD: actinomycin D; CHL: classical Hodgkin lymphoma; CHX: cycloheximide; EBV: Epstein-Barr virus; MEL: melanoma; Monos: monocytes; NSCLC: nonsmall-cell lung carcinoma; PD-L1: programmed death-ligand 1; qRTPCR: quantitative reverse transcriptase polymerase chain reaction; RCC: renal cell carcinoma; SCCHN: squamous cell carcinoma of head and neck; STAT: Signal transducer and activator of transcription; TME: tumor microenvironment

\section{Acknowledgements}

The authors thank Hao Wang for advice on statistical analyses, Paige Damascus for technical support, Chirag Patel for advice on siRNA transfection procedures and blocking mRNA and protein synthesis in vitro, and Daria Gaykalova for providing SCCHN cell lines (all from Johns Hopkins University School of Medicine, Baltimore, MD); and James C. Yang (National Institutes of Health, Bethesda, MD) for providing RCC cell lines.

\section{Authors' contributions}

SC designed, conducted and analyzed experiments, and wrote the manuscript. GAC, TSP, TLM and PW acquired and analyzed data. DMP and FP supervised and analyzed portions of this study. SLT designed and supervised the study and co-wrote the manuscript. All authors reviewed and approved the manuscript.

\section{Funding}

This study was supported by NCI R01 CA142779 (DMP and SLT), the Barney Family Foundation (SLT), Moving for Melanoma of Delaware (SLT), the Laverna Hahn Charitable Trust (SLT), R01 Al089830 (FP), Melanoma Research Alliance (FP) and the Johns Hopkins Bloomberg Kimmel Institute for Cancer Immunotherapy.

\section{Availability of data and materials}

All data generated or analyzed during this study are included in this published article and its supplementary information files.

\section{Ethics approval and consent to participate}

The use of human tissues in this study was approved by the Johns Hopkins Institutional Review Board.

\section{Consent for publication}

Not applicable.

\section{Competing interests}

DMP and SLT report stock and other ownership interests from Aduro Biotech, Compugen, DNAtrix, Dragonfly Therapeutics, ERVAXX, Five Prime Therapeutics, FLX Bio, Jounce Therapeutics, Potenza Therapeutics, Tizona Therapeutics, and WindMIL; consulting or advisory role with AbbVie, Amgen, Bayer, Compugen, DNAtrix, Dragonfly Therapeutics, Dynavax, ERVAXX, Five Prime Therapeutics, FLX Bio, Immunomic Therapeutics, Janssen Oncology, Medlmmune, Merck, Tizona Therapeutics, and Wind MIL; research funding from Bristol-Myers Squibb, Compugen, and Potenza Therapeutics; patents, royalties, and other intellectual property from Aduro Biotech, Bristol-Myers Squibb, and Immunonomic Therapeutics; and travel, accommodations, expenses from Bristol-Myers Squibb, and Five Prime Therapeutics. SC, GAC, TSP, PW, TLM and FP have no conflicts of interest to disclose.

\section{Author details}

'Department of Surgery, Johns Hopkins University School of Medicine, Sidney Kimmel Comprehensive Cancer Center, and Bloomberg Kimmel Institute for Cancer Immunotherapy, Baltimore, MD 21287, USA. ${ }^{2}$ Department of Oncology, Johns Hopkins University School of Medicine, Sidney Kimmel Comprehensive Cancer Center, and Bloomberg Kimmel Institute for Cancer Immunotherapy, Baltimore, MD 21287, USA.

Received: 2 July 2019 Accepted: 2 October 2019

Published online: 15 November 2019

\section{References}

1. Topalian SL, Weiner GJ, Pardoll DM. Cancer immunotherapy comes of age. J Clin Oncol. 2011;29(36):4828-36. 
2. Dong H, Strome SE, Salomao DR, Tamura H, Hirano F, Flies DB, et al. Tumorassociated B7-H1 promotes T-cell apoptosis: a potential mechanism of immune evasion. Nat Med. 2002;8(8):793-800.

3. Topalian SL, Taube JM, Anders RA, Pardoll DM. Mechanism-driven biomarkers to guide immune checkpoint blockade in cancer therapy. Nat Rev Cancer. 2016;16(5):275-87.

4. Pardoll DM. The blockade of immune checkpoints in cancer immunotherapy. Nat Rev Cancer. 2012;12(4):252-64.

5. Marzec M, Zhang Q, Goradia A, Raghunath PN, Liu X, Paessler M, et al. Oncogenic kinase NPM/ALK induces through STAT3 expression of immunosuppressive protein CD274 (PD-L1, B7-H1). Proc Natl Acad Sci U S A. 2008;105(52):20852-7.

6. Green MR, Rodig S, Juszczynski P, Ouyang J, Sinha P, O'Donnell E, et al. Constitutive AP-1 activity and EBV infection induce PD-L1 in Hodgkin lymphomas and posttransplant lymphoproliferative disorders: implications for targeted therapy. Clin Cancer Res. 2012;18(6):1611-8.

7. Chen L, Gibbons DL, Goswami S, Cortez MA, Ahn YH, Byers LA, et al. Metastasis is regulated via microRNA-200/ZEB1 axis control of tumour cell PD-L1 expression and intratumoral immunosuppression. Nat Commun. 2014,5:5241

8. Jiang X, Zhou J, Giobbie-Hurder A, Wargo J, Hodi FS. The activation of MAPK in melanoma cells resistant to BRAF inhibition promotes PD-L1 expression that is reversible by MEK and PI3K inhibition. Clin Cancer Res. 2013;19(3):598-609.

9. Crane C, Panner A, Pieper RO, Arbiser J, Parsa AT. Honokiol-mediated inhibition of PI3K/mTOR pathway: a potential strategy to overcome immunoresistance in glioma, breast, and prostate carcinoma without impacting T cell function. J Immunother. 2009;32(6):585-92.

10. Casey SC, Tong L, Li Y, Do R, Walz S, Fitzgerald KN, et al. MYC regulates the antitumor immune response through CD47 and PD-L1. Science. 2016; 352(6282):227-31.

11. Zhang J, Bu X, Wang H, Zhu Y, Geng Y, Nihira NT, et al. Cyclin D-CDK4 kinase destabilizes PD-L1 via cullin 3-SPOP to control cancer immune surveillance. Nature. 2018;553(7686):91-5.

12. Li CW, Lim SO, Xia W, Lee HH, Chan LC, Kuo CW, et al. Glycosylation and stabilization of programmed death ligand-1 suppresses T-cell activity. Nat Commun. 2016;7:12632.

13. Taube JM, Young GD, McMiller TL, Chen S, Salas JT, Pritchard TS, et al. Differential expression of immune-regulatory genes associated with PD-L1 display in melanoma: implications for PD-1 pathway blockade. Clin Cancer Res. 2015;21(17):3969-76.

14. Duffield AS, Ascierto ML, Anders RA, Taube JM, Meeker AK, Chen S, et al. Th17 immune microenvironment in Epstein-Barr virus-negative Hodgkin lymphoma: implications for immunotherapy. Blood Adv. 2017; 1(17):1324-34

15. Taube JM, Anders RA, Young GD, Xu H, Sharma R, McMiller TL, et al. Colocalization of inflammatory response with B7-h1 expression in human melanocytic lesions supports an adaptive resistance mechanism of immune escape. Sci Transl Med. 2012;4(127):127ra37.

16. Carbotti G, Barisione G, Airoldi I, Mezzanzanica D, Bagnoli M, Ferrero S, et al. IL-27 induces the expression of IDO and PD-L1 in human cancer cells. Oncotarget. 2015;6(41):43267-80.

17. Bellucci R, Martin A, Bommarito D, Wang K, Hansen SH, Freeman GJ, et al. Interferon-gamma-induced activation of JAK1 and JAK2 suppresses tumor cell susceptibility to NK cells through upregulation of PD-L1 expression. Oncoimmunology. 2015;4(6):e1008824.

18. Lee SJ, Jang BC, Lee SW, Yang YI, Suh SI, Park YM, et al. Interferon regulatory factor-1 is prerequisite to the constitutive expression and IFN-gammainduced upregulation of B7-H1 (CD274). FEBS Lett. 2006;580(3):755-62.

19. Gowrishankar K, Gunatilake D, Gallagher SJ, Tiffen J, Rizos H, Hersey P. Inducible but not constitutive expression of PD-L1 in human melanoma cells is dependent on activation of NF-kappaB. PLoS One. 2015;10(4): e0123410

20. Dorand RD, Nthale J, Myers JT, Barkauskas DS, Avril S, Chirieleison SM, et al. Cdk5 disruption attenuates tumor PD-L1 expression and promotes antitumor immunity. Science. 2016;353(6297):399-403.

21. NIH. ImageJ. https://imagej.nih.gov/ij/ (2004).

22. Lonza Knowledge Center. https://knowledge.lonza.com/ (2016).

23. Zaretsky JM, Garcia-Diaz A, Shin DS, Escuin-Ordinas H, Hugo W, HuLieskovan S, et al. Mutations associated with acquired resistance to PD-1 blockade in melanoma. N Engl J Med. 2016;375(9):819-29.
24. Shuai K, Schindler C, Prezioso VR, Darnell JE Jr. Activation of transcription by IFN-gamma: tyrosine phosphorylation of a 91-kD DNA binding protein. Science. 1992;258(5089):1808-12.

25. Caldenhoven E, Buitenhuis M, van Dijk TB, Raaijmakers JA, Lammers JW, Koenderman L, et al. Lineage-specific activation of STAT3 by interferongamma in human neutrophils. J Leukoc Biol. 1999;65(3):391-6.

26. Weber A, Wasiliew P, Kracht M. Interleukin-1 (IL-1) pathway. Sci Signal. 2010; 3(105): $\mathrm{cm} 1$.

27. Hirahara K, Ghoreschi K, Yang XP, Takahashi H, Laurence A, Vahedi G, et al Interleukin-27 priming of T cells controls IL-17 production in trans via induction of the ligand PD-L1. Immunity. 2012;36(6):1017-30.

28. NIH. dbSNP. https://www.ncbi.nlm.nih.gov/snp (2018).

29. Cottrell TR, Taube JM. PD-L1 and emerging biomarkers in immune checkpoint blockade therapy. Cancer J. 2018;24(1):41-6.

30. Cerezo M, Guemiri R, Druillennec S, Girault I, Malka-Mahieu H, Shen S, et al. Translational control of tumor immune escape via the elF4F-STAT1-PD-L1 axis in melanoma. Nat Med. 2018;24(12):1877-86.

31. Johnson DE, O'Keefe RA, Grandis JR. Targeting the IL-6/JAK/STAT3 signalling axis in cancer. Nat Rev Clin Oncol. 2018;15(4):234-48.

32. Dinarello CA, Simon A, van der Meer JW. Treating inflammation by blocking interleukin-1 in a broad spectrum of diseases. Nat Rev Drug Discov. 2012; 11(8):633-52.

\section{Publisher's Note}

Springer Nature remains neutral with regard to jurisdictional claims in published maps and institutional affiliations.

Ready to submit your research? Choose BMC and benefit from:

- fast, convenient online submission

- thorough peer review by experienced researchers in your field

- rapid publication on acceptance

- support for research data, including large and complex data types

- gold Open Access which fosters wider collaboration and increased citations

- maximum visibility for your research: over $100 \mathrm{M}$ website views per year

At BMC, research is always in progress.

Learn more biomedcentral.com/submission 